\title{
The Intelligent Supervision of The Agriculture Production Based on Internet of Things and Cloud Service Platform
}

\author{
Chaodong Miao \\ Yixing Secondary Vocational School of Jiangsu Province, Yixing 214206, China
}

Keywords: cloud service platform, agriculture production, the internet of things.

\begin{abstract}
Aiming to the precision, information and intelligence development requirements of modern agriculture, the scheme of environmental parameters monitoring and automatic control for agriculture production based on cloud computation and internet of things is put up. Through sensors, zigbee wireless network, internet and GPRS technology, the system realize the real-time monitoring the luminance, temperature and humidity of air, concentration of $\mathrm{CO}_{2}$, temperature and humidity of soil, soil PH value, meanwhile, the different devices are controlled to regulate the environmental parameters. The Zigbee modules are used to build up wireless network, and the cloud service platform is connected by remote gateway through TCP/IP protocol and GPRS mobile network, the information can be transferred up and down. The cloud service platform can provide data for web browser and App terminal.
\end{abstract}

\section{Introduction}

Traditional agriculture production relies on the farmer's experience to cultivate the plants. But in traditional agriculture production, the growing environment cannot be controlled precisely. The modern agriculture is characterized by precision management, information sharing and intelligence control[1-3]. The internet of things (IoT) including sensors, sensor network, internet, mobile internet, and cloud computation can pay an import role in the modern agriculture. Through the internet of things technology, the followings can be realized, including the intelligent supervision of agriculture production, quality tracing of agricultural products, the informationization of agricultural products trade. In recent years, the intelligent supervision of agriculture production based on IoT technology is applied in vegetable planting, grape planting, which promotes the management efficiency of the agriculture production, raises the output rate of the agriculture production, improves the ecological environment of agriculture production. The intelligent supervision of agriculture production is a closed loop control system. Different types of sensors can detect the environment parameters of the agriculture production, the sensor data can be transmitted to the cloud service platform by the self-organized wireless sensor network through the wireless gateway. The software decision support system can make the control decision according to the control algorithm, and the control command can be sent to the actuator and related devices can be controlled. Combined the cloud computation and the IoT technology, characterized by environmental sensor, device controlled, remote transmission, and cloud service platform, the intelligent supervision of the agriculture production has been applied in the agriculture ecological gardens located in Anhui province and Shanghai City.

\section{The general system architecture}

The intelligent supervision of the agriculture production is comprised by sensor data acquisition system, environment control system, wireless sensor network based on Zigbee, remote gateway, cloud service platform. The system architecture can be seen in Fig.1. The sensor data acquisition system is mainly responsible for obtaining the five kinds of environment data. Environment control system is mainly responsible for receiving the instructions of the cloud service platform, and realize the function of ventilation fan, cooling device, heating device, light supplement, and sun shading. The sensor is connected with RS232 corresponding port, and the sensor data is transmitted to the Zigbee node by RS232 port, The star wireless local network can be comprised by several zigbee terminal 
nodes and the coordinator nodes. The coordinator nodes can correspond with the remote gateway by RS32. The remote gateway can send the data remotely to the cloud service platform through TCP/IP protocol or GPRS protocol. The measuring data is sent to the cloud service platform based on Internet network and mobile networkl. The cloud service platform sends the data acquisition instruction to the central data acquisition treatment device, which direct the sensor to acquisition the data.

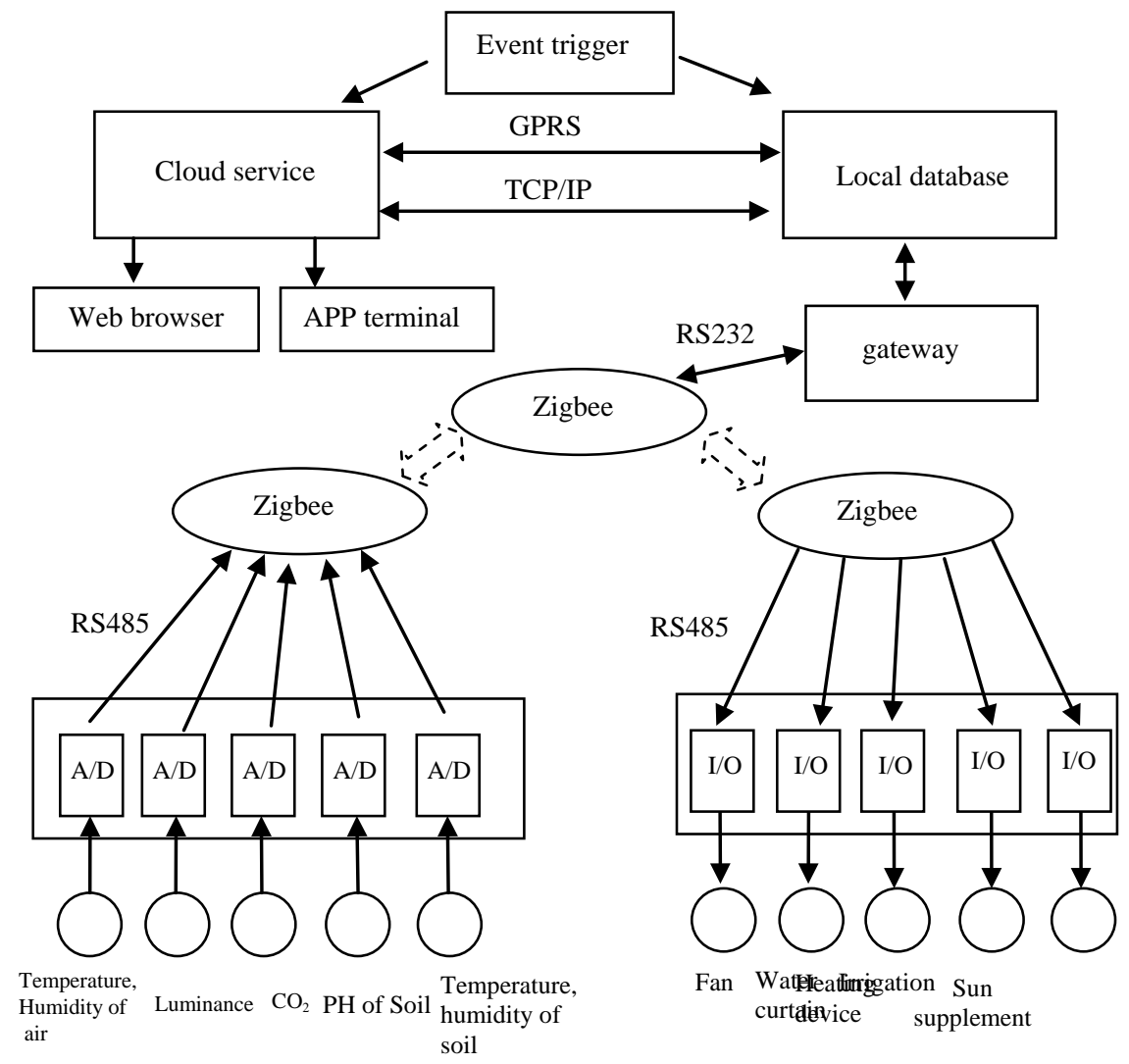

Fig.1 System architecture

\section{Data acquisition and environment control for agriculture IoT}

Five kinds of parameters are collected in agriculture production, including luminance, temperature and humidity of air, concentration of $\mathrm{CO}_{2}$, soil moisture, and $\mathrm{PH}$ value of soil. The signals of the sensors are voltage or current, the voltage scope is $0-3.3 \mathrm{~V}$, and the current signal is $4-20 \mathrm{~mA}$ 。To achieve the environmental control, according to the growing condition of the crops, the cloud service platform can control the ventilation machine, screen spray, supplement lamp, heating device, $\mathrm{CO}_{2}$ generator and automatic device for irrigation through web software or mobile APP software.

\section{Luminance sensor}

To measure the sun light luminance, the luminance sensor TSL 2560 is used, which has high sensitivity for low light condition. This sensor has the following advantage, has wide measuring scope, good linearity, good water proof, and easy usage, and convenient installation. TSL 2560 sensor is applicable for agriculture greenhouse. The input voltage of the sensor is between $2.5 \mathrm{~V}$ to $5.5 \mathrm{~V}$, and the measuring scope of the light luminance is 0 to 38000 Lux. At low energy consumption mode, the power is only $0.75 \mathrm{~mW}$.

\section{The temperature and humidity sensor}

The SHT10 humidity and temperature sensor is a kind of digital temperature and humidity sensor chip which is made by Swiss corporation Sensirion. This kind of sensor has the advantage of high integration, low power consumption, high precision, and small size. The SHT10 chip has been integrated 14 bit AD converter, and the output is digital signal, has high reliability and long lifetime. 


\section{$\mathrm{CO}_{2}$ sensor}

The $\mathrm{CO}_{2}$ sensor is PY3228TC G2/G20 type, which is made by Perkinelmer company, has two channel with temperature compensation, one channel is reference channel, and the other channel is the measuring channel. The solid photoelectric sensor is used the NDIR infra-red technique, when the $\mathrm{CO}_{2}$ concentration is measured, the temperature is compensated, which is suitable for measuring the temperature and $\mathrm{CO}_{2}$ concentration at the same time.

\section{PH sensor for soil}

$\mathrm{PH}$ sensor for soil uses the solid electrolyte, pressure endurance, not easy to block, can on-line detecting for long time. The electrode can use different sensitive membrane electrode according to the different using condition and detect the $\mathrm{PH}$ value.

\section{Water content sensor for soil}

5TM Water content sensor is made by American Decgon. The principle of the 5TM water content sensor is based on the dielectric theory, which is frequency domain reflectometry. The difference of dielectric constant is big between the different substance, so in the soil, the dielectric constant of the air and other substances is far smaller than the water, so neglecting the effect of the other substance, the dielectric constant is mainly determined by the water content of the soil.

\section{Power supply for sensor}

The solar power system supplies the power of the sensors. The solar panel is $12 \mathrm{~V} / 30 \mathrm{~W}$ with the area of $500 \times 600 \mathrm{~mm}$, which connected with the storage battery. The life time is five years long, the working endurance is not less than six days. To supply the power for the luminance sensor, temperature and humidity sensor, $\mathrm{CO}_{2}$ sensor, $\mathrm{PH}$ sensor, and soil moisture sensor, six solar panels and two 200AH storage battery are used.

\section{The architecture of the cloud service platform}

The cloud service platform is constituted by one network load balancer, two firewall and two three-tier switches, which promote the redundancy, prevent single point of failure. Two high performance HP servers connect with Hitachi HUS110 high performance storage through two optical switches. When the main database server is down, the other standby server can be activated immediately, which takes over the data application service and make the cloud service platform can run smoothly. After the main server recovery from failure, the system returns to the normal state. The separate backup server with backup software can continuously backup the database file to the specific storage service.

\section{Application software}

The IoT system for agriculture is integrated the Internet with wireless sensor network, which supply the intelligent service for the farmers. With the development of cloud computation technology, the service abilities can be promoted remarkably. The cloud service platform is based on AJAX technology, and the application structure is B/S browns. The client server is based on PHP technology, the server database is SQL Server2012. Through IIS, the Web service is published. This kind of structure decreases the transmit data volume and increases the show velocity of the web browser.

\section{The field application}

The IoT system for agriculture can realize the precision management for the agriculture production, which supply the powerful technology support for the administrative staff, people in the front line of agriculture production. The function of the system includes automation alert, automation control, data acquisition, data transmission. The system has been successfully applied in greenhouse in Ningguo City, Anhui province, China, See Fig.2. 


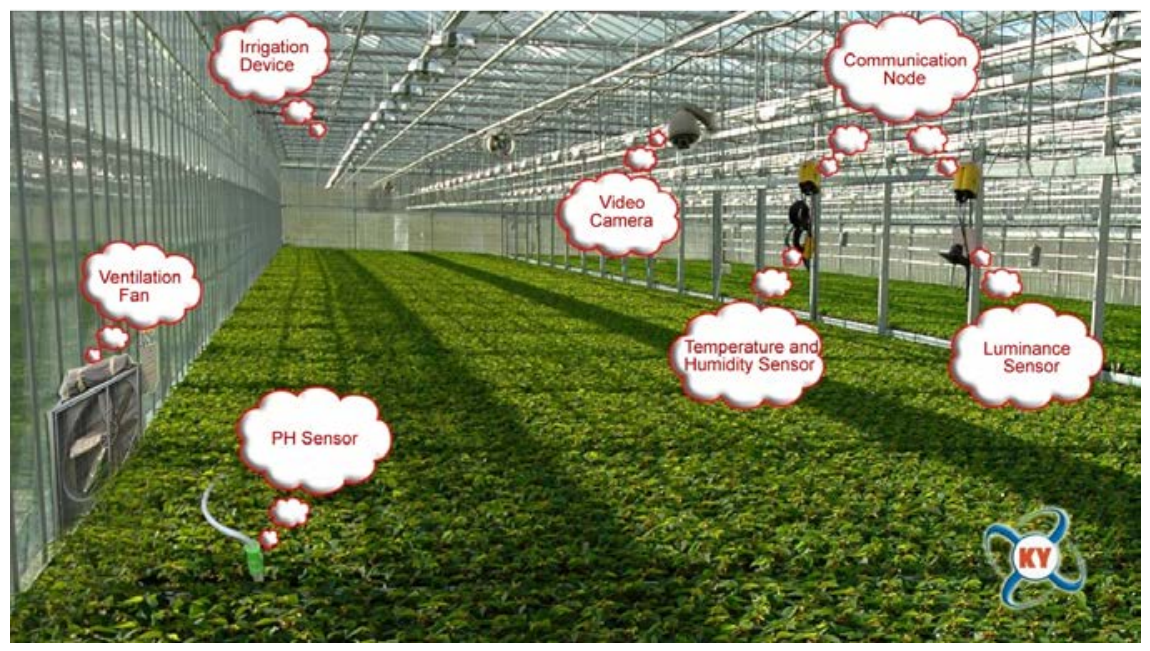

Fig.2 Greenhouse in Ningguo City, Anhui

\section{Conclusion}

The sensors for measuring the luminance, temperature and humidity of air, concentration of CO2, soil moisture, and PH value of soil have been used in the system. The greenhouse environment sensor data are transmitted to the background system through the wireless nodes. The data can be output as the graphical display after treatment by the management software. If the index is exceed the set point, the system can output the alert signal, at the same time, the ventilation fan, electrical valve, sun shelt can be open and shut intelligently. The system is suitable for the remote supervision of the agriculture production. The system is an attempt on using the cloud computation, mobile network, Internet to the agriculture. The field application shows that the system is stable and economical. Meanwhile, the system is easy to upgrade and reconfigure.

\section{Acknowledgements}

This research was a project of our cooperation with Anhui in Ningguo City.

\section{References}

[1] Y. Kim, R.G. Evans, W. M. Iversen, Remote sensing and control of an irrigation system using a distributed wireless sensor network. IEEE Transactions on Instrument, 2008, 57(7): 1379-1387

[2] J. A. Jiang, T.S. Lin, E.C. Yang, etal., Application of web-based remote agro-ecological monitoring system for observing spatial distribution and dynamics of Bactrocera dorsalis in fruit orchards. Precision Agric., 2013, 14: 323-342

[3] H. Zhou, B. W. Liu, P.P. Dong, The technology system framework of the internet of things and its application research in agriculture, in: D. Li, Y. Chen (Eds.), CCTA 2011, Part I, IFIP AICT,2012,pp.293-300 